\title{
Could We Use Vital Signs and Lactate Levels Together to Predict the Prognosis in Abdominal Pain?
}

\author{
Filiz Froohari Damarsoy', [MD] \\ ORCID: 0000-0002-5444-1288 \\ Nalan Metin Aksu' ${ }^{1}$, [MD] \\ ORCID: 0000-0003-2256-5187 \\ Elif Öztürk' , [MD] \\ ORCID: 0000-0002-6396-6668 \\ Meltem Akkaş ${ }^{1}$, [MD] \\ ORCID: 0000-0003-1582-9631
}

'Hacettepe University, Faculty of Medicine, Department of Emergency Medicine, Ankara/Turkey.

Corresponding Author: Filiz Froohari Damarsoy Hacettepe University, Faculty of Medicine, Department of Emergency Medicine, Ankara/Turkey.

E-mail: filizfroohari@gmail.com

https://doi.org/10.32552/2021.ActaMedica.634

\section{ree) ABSTRACT Cere}

Objectives: Abdominal pain occupies most of the emergency department admissions. This entity leads to research various markers for the early detection of causes in patients presenting with abdominal pain. There is limited data about collaborations between lactate levels and vital signs at admission in abdominal pain.

Materials and Methods: Patients aged 18 years and older, who had presented with abdominal pain to the Emergency Department, in total 102 patients, were included to the study. The patients' demographics, vital signs, abdominal physical examination findings, diagnosis and outcomes of patients were recorded. The $1 \mathrm{ml}$ venous blood samples were collected by blood gase injectors from the patients and lactate levels were analyzed.

Results: Female patients' percentile was $68,6 \%$ and mean age was 39 years old. The systolic blood pressure levels were normal in 45 patients, low in $26 \%$ patients and high in $29 \%$ patients. The seventy-six of the patients had normal pulse rate and 26 of them were tachycardic. Most common diagnosis was non-specific abdominal pain $(37,25 \%)$ and the least common was ovarian torsion/intracystic hemorrhage $(0,98 \%)$ and obstruction due to hernia $(0,98 \%)$. There was no statistically significant relation between lactate level and pulse rate $(p=0,637)$, systolic blood pressure $(p=0,052)$, diastolic blood pressure $(p=0,095)$, respiratory rate $(p=0,527)$, body temperature $(p=0,040)$ and oxygen saturation $(p=0,905)$. Similarly, no significant association occurred between lactate levels and diagnosis and outcomes.

Conclusion: Further studies including more patients groups have to be done in order to attain more reliable data about this topic.

Keywords: Abdominal pain, vital signs, lactate, emergency department

Received: 9 June 2021, Accepted: 3 August 2021,

Published online: 20 November 2021 


\section{INTRODUCTION}

The causes of abdominal pain constitute a spectrum that ranges from acute and life-threatening emergencies. It can be challenging to diagnose and treat the underlying causes of abdominal pain, and advanced examinations are often necessary. In this regard, clinicians sometimes perform unnecessary procedures when they cannot otherwise find the cause of the pain.

Relatedly, investigators are still reviewing the hospitalization criteria, as well as various markers, for patients presenting with abdominal pain. In the present study specifically, we used a lactate test, which can be performed using a venous blood gas injector. We hypothesized that If the lactate level is above the established laboratory threshold, the treatment will be started immediately without detailed diagnostic procedures.

\section{MATERIALS and METHODS}

\section{Patients}

The local ethical committee approved this prospective observational study (16969557/1228). Patients aged 18 years and older, who had presented with abdominal pain to the Emergency Department (ED) in a 6-month period, in total 102 patients, were included to the study. All patients, or their relatives, signed written consent forms. The patients' demographic characteristics, vital signs, abdominal physical examination findings, the diagnosis and outcomes were recorded.

We evaluated and recorded-at the time of first presentation to the ED- blood pressure (BP), heart rate $(H R)$, respiratory rate $(\mathrm{RR})$, body temperature, and oxygen saturation ( $\mathrm{SaO} 2)$. Only patients with a Glasgow Coma Scale (GCS) score of 15 were included in the study. To analyze BP, we classified the patients into 3 groups on the basis of the systolic BP value: "hypotensive" $(<90 \mathrm{mmHg})$,"normotensive" (90-140 $\mathrm{mmHg}$ ), and "hypertensive" (> $140 \mathrm{mmHg}$ ). Heart rate were classified as "bradycardia" ( $<60$ beats/ min), "normal" (60-99 beats/min), and "tachycardia" ( $\geq 100$ beats/min). Hypoxia was defined as an $\mathrm{SaO} 2$ of $<94 \%$. With regard to the bowel sounds heard during auscultation, 4-10 sounds per minute was considered the normal range. The $1 \mathrm{ml}$ venous blood samples were collected in blood gauze injectors from the patients and lactate levels were analyzed in our hospital Biochemistry Laboratory. The ranges of the lactete levels were accepted as $0.9-1.7 \mathrm{mmol} / \mathrm{L}$ in our hospital laboratory. We then evaluated the lactate level in terms of three groups: "low", "normal", and "high" lactate.

\section{Statistical Methods}

Statistical analyses were performed using the IBM SPSS for Windows Version 21.0 software package. Numerical variables were expressed as mean \pm standard deviation, while categorical variables were expressed as numbers and percentages. Differences between groups were analyzed using the chi-square test or Fisher's exact test, and $p$-values $<0.05$ were considered statistically significant.

\section{Inclusion and Exclusion Criteria}

The inclusion criteria were as following; patients had to be (1) aged $\geq 18$ year who had presented to the ED complaining of abdominal pain; (2) willing to participate in the study, having read and signed the consent form; (3) cooperative; and (4) oriented (with a GCS score of 15). Both men and women were recruited in this study.

The exclusion criteria were as following: (1) dyspnea, respiratory distress, or similar (patients with chronic obstructive pulmonary disease, asthma, congestive heart failure, etc. were included, provided they did not display the symptoms mentioned); (2) chronic liver disease; (3) sepsis or septic shock; (4) hemorrhagic shock; (5) cardiogenic shock; (6) any neoplastic disease; (7) trauma; (8) pregnancy; (9) drug intoxication; or (10) hypoxia. We also excluded patients who were using drugs that lead to lactic acidosis (such as metformin), who had undergone gastric bypass surgery at any time, who had performed strenuous exercise or sport activities recently, and who had a history of alcoholism.

\section{RESULTS}

Of the 102 patients included in the study, 32 (31.3\%) were men and 70 (68.6\%) were women. The mean age of the patients was $38.59 \pm 18.02$ years (range: 18-90 years). Table 1 shows the distribution 
of patients according to the vital signs at the time of admission, while Table 2 shows the distribution of patients according to the examination findings. The distribution of the patients according to the diagnosis was illustrated in Figure 1. We examined whether the serum lactate levels of all the patients were associated with various other parameters. In terms of vital signs, as measured at triage, no significant association was found between lactate level and heart rate $(p=0.637)$, systolic blood pressure $(p=0.052)$, diastolic blood pressure $(p=0.095)$, respiratory rate $(p=0.527)$, body temperature $(p=0.040)$, oxygen saturation $(p=$ 0.905). Table 3 shows the distribution of lactate levels in terms of vital signs.

With regard to whether lactate levels were associated with abdominal examination findings, no significant association was observed between lactate levels and rebound, defense, costovertebral angle tenderness (CVAT), and bowel sounds (Table 4).

Similarly, no significant association was detected between lactate levels and diagnosis and outcomes (Table 5).

\section{DISCUSSION}

When patients present to the ED with abdominal pain, the clinician should first carry out an in-depth evaluation of the anamnesis, as well as a physical examination. In light of this information, a table of pre-diagnoses should be produced, and the available findings should be examined thoroughly, beginning with the most suspected pre-diagnoses. In this way, the diagnosis can be established by using shortcuts; such an approach prevents the patient's and clinician's time from being wasted.

Of the 102 patients included in our study, 70 were women (68.6\%) and 32 (31.4\%) were men. Their ages ranged from 18 to 90 years, with a mean age of $38.59 \pm 18.02$ years. These findings were similar to the studies about this topic [1-3].

In a study by Çalışkan et al. [4], the pulse values of 957 patients who had presented to the ED with acute abdominal pain ranged from 54 to 110 beats/ $\mathrm{min}$, and the mean pulse value was $77.61 \pm 7.08$ beats $/ \mathrm{min}$. The pulse values of $28.6 \%$ of the patients diagnosed with perforated appendicitis, and 12\% of the patients diagnosed with acute appendicitis,

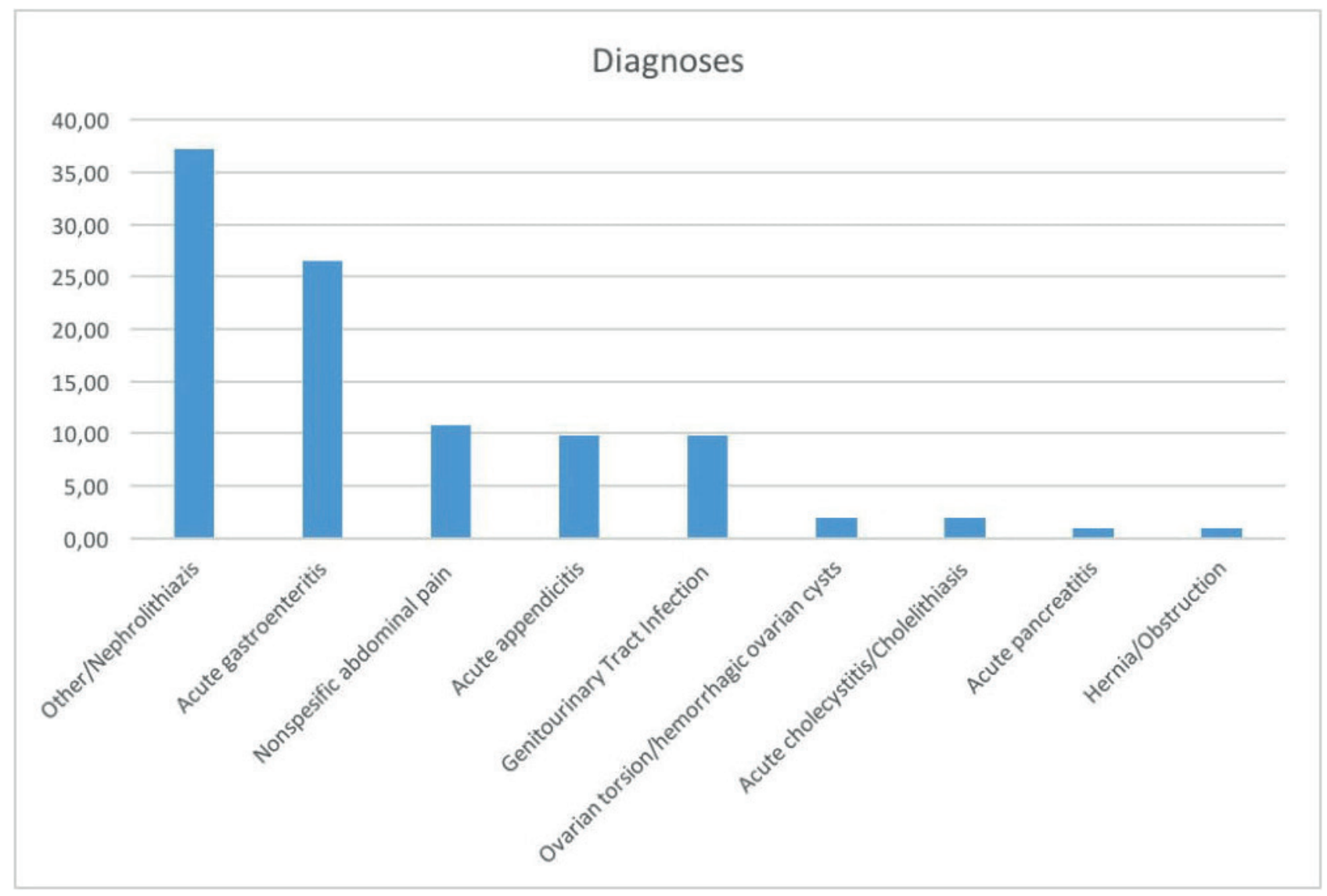

Figure 1. The distribution of the patients according to the diagnosis 
Table 1. The distribution of the patients due to their vital signs

\begin{tabular}{|l|c|c|c|c|c|c|}
\hline \multirow{2}{*}{} & \multicolumn{2}{|c|}{ Normal } & \multicolumn{2}{c|}{ Low } & \multicolumn{2}{c|}{ High } \\
\cline { 2 - 7 } & (n) & $\%$ & (n) & $\%$ & (n) & \% \\
\hline Blood Pressure & 45 & 44.1 & 28 & 27.5 & 26 & 28.4 \\
Heart Rate & 76 & 74.5 & 0 & 0 & 0 & 0 \\
O2 Sat. & 98 & 96.1 & 4 & 0 & 0 & 0 \\
\hline GKS & 102 & 0 & 0 & 0.9 & 0 \\
\hline
\end{tabular}

Table 2. The distribution of the patients due to their abdominal examination findings

\begin{tabular}{|l|c|c|}
\hline \multirow{2}{*}{} & \multicolumn{2}{|c|}{ Patients } \\
\cline { 2 - 3 } & Positive & Negative \\
\hline Rebound & $\mathbf{N}(\%)$ & $93(91.2)$ \\
Defans & $9(8.8)$ & $97(95.1)$ \\
Costovertebral angle tenderness & $5(4.9)$ & $89(87.3)$ \\
Assit & $13(12.7)$ & $102(100)$ \\
Icterus & $0(0)$ & $102(100)$ \\
Visible mass & $0(0)$ & $102(100)$ \\
Increased bowel sounds & $0(0)$ & $89(87.3)$ \\
\hline
\end{tabular}

Table 3. The distribution of the patients due to the relationship between vital signs and lactate levels

\begin{tabular}{|c|c|c|c|c|c|}
\hline \multirow{2}{*}{\multicolumn{2}{|c|}{ Vital Signs }} & \multicolumn{4}{|c|}{ Lactate Levels } \\
\hline & & \multirow{2}{*}{$\begin{array}{c}\text { Normal } \\
39\end{array}$} & \multirow{2}{*}{$\begin{array}{c}\text { Low } \\
12\end{array}$} & \multirow{2}{*}{$\begin{array}{c}\text { High } \\
15\end{array}$} & $p$ \\
\hline \multirow{3}{*}{ Heart rate } & Normal & & & & \multirow{3}{*}{0,637} \\
\hline & Low & 0 & 0 & 0 & \\
\hline & High & 15 & 5 & 6 & \\
\hline \multirow{3}{*}{ Systolic BP } & Normal & 28 & 6 & 11 & \multirow{3}{*}{0,052} \\
\hline & Low & 10 & 9 & 9 & \\
\hline & High & 16 & 2 & 11 & \\
\hline \multirow{3}{*}{ Diastolic BP } & Normal & 27 & 6 & 15 & \multirow{3}{*}{0,095} \\
\hline & Low & 8 & 8 & 7 & \\
\hline & High & 19 & 3 & 9 & \\
\hline \multirow{2}{*}{$\mathrm{O}_{2}$ sat } & Normal & 52 & 16 & 30 & \multirow{2}{*}{0,905} \\
\hline & Low & 2 & 1 & 1 & \\
\hline
\end{tabular}

Table 4. The distribution of the patients due to the relationship between abdominal examination findings and lactate levels

\begin{tabular}{|c|c|c|c|c|c|}
\hline \multirow{2}{*}{\multicolumn{2}{|c|}{ Abdominal examination findings }} & \multicolumn{4}{|c|}{ Lactate levels } \\
\hline & & \multirow{2}{*}{$\begin{array}{c}\text { Normal(n) } \\
6\end{array}$} & \multirow{2}{*}{$\begin{array}{c}\operatorname{Low}(\mathrm{n}) \\
2\end{array}$} & \multirow{2}{*}{$\begin{array}{c}\operatorname{High}(\mathrm{n}) \\
1\end{array}$} & $\mathbf{p}$ \\
\hline \multirow{2}{*}{ Rebound } & Positive & & & & \multirow{2}{*}{0,358} \\
\hline & Negative & 48 & 15 & 30 & \\
\hline \multirow{2}{*}{ Defans } & Positive & 3 & 1 & 1 & \multirow{2}{*}{0,864} \\
\hline & Negative & 51 & 16 & 30 & \\
\hline \multirow{2}{*}{ Costovertebral angle tenderness } & Positive & 9 & 1 & 1 & \multirow{2}{*}{0,397} \\
\hline & Negative & 45 & 16 & 28 & \\
\hline \multirow{3}{*}{ Bowel sounds } & Normal & 47 & 15 & 27 & \multirow{3}{*}{0,632} \\
\hline & Low & 0 & 0 & 1 & \\
\hline & High & 7 & 2 & 3 & \\
\hline
\end{tabular}


Table 5. The distribution of the patients due to the relation between diagnoses, outcomes and lactate level

\begin{tabular}{|c|c|c|c|c|c|}
\hline & & \multicolumn{4}{|c|}{ Lactate Levels } \\
\hline & & Normal(n) & $\operatorname{Low}(\mathrm{n})$ & $\operatorname{High}(\mathrm{n})$ & $\mathbf{P}$ \\
\hline \multirow{9}{*}{ Diagnoses } & Nonspecific abdominal pain & 5 & 3 & 3 & \multirow{9}{*}{0.381} \\
\hline & Cholelatiasis/cholesysit & 0 & 0 & 2 & \\
\hline & Acute pancreatitis & 0 & 0 & 1 & \\
\hline & Acute appendisite & 8 & 1 & 1 & \\
\hline & Ovarian torsion & 1 & 1 & 0 & \\
\hline & Hernia/Obstruction & 0 & 0 & 1 & \\
\hline & Other/ Nephro/urolithiasis & 18 & 6 & 14 & \\
\hline & Gastroenterit & 16 & 3 & 8 & \\
\hline & Urinary system infections & 6 & 3 & 1 & \\
\hline \multirow{4}{*}{ Outcomes } & Discharged & 43 & 15 & 26 & \multirow{4}{*}{0.381} \\
\hline & Discharged against medical advice & 0 & 0 & 1 & \\
\hline & Left without permission & 1 & 0 & 0 & \\
\hline & Hospitalized & 11 & 2 & 3 & \\
\hline
\end{tabular}

were deemed to be high in the same study [4], body temperature measurements and pulse values were given in tables. Their results were similar to our own in terms of pulse value; however, 10 of their patients were diagnosed with acute appendicitis, and those patients had no tachycardia.

In our study, all patients showed abdominal tenderness. Among them, 9 (8.8\%) showed rebound, 5 (4.9\%) defense, and 13 (12.7\%) CVAT. Furthermore, 12 (11.8\%) patients showed increased bowel sounds, and 1 had a mechanical bowel sound. In a study by Chen et al (3), 20 (19\%) of 309 patients had no tenderness, 163 had tenderness, and 9 (24\%) had rebound. Among the 166 patients included in a study by Yeniocak et al. [5], 72 (43.4\%) only showed abdominal tenderness as a physical examination finding, 36 (21.7\%) showed concomitant defense, 7 (4.2\%) showed rebound, and 14 (8.49\%) showed both defense and rebound.

In the study by Çalışkan et al. [4], 628 (65.6\%) patients had bowel sounds within normal limits, $231(24.1 \%)$ had increased bowel sounds, and 98 (10.2\%) had decreased bowel sounds. There were $948(99.1 \%)$ patients with abdominal tenderness, 244 (25.5\%) with defense, and 146 (15.3\%) with rebound. Nagurney et al. (2) found that 24 patients (19\%) had extensive abdominal tenderness in all quadrants, and that 18 (15\%) had CVAT. We couldn't have encountered study that searching the relationship between abdominal pain and icterus, dullness, or ascites. In the study by Çalışkan et al. [4], $32 \%$ of patients diagnosed with acute appendicitis showed no defense, and $32 \%$ showed no rebound; $63.6 \%$ of patients with acute pancreatitis showed no defense, and $90.9 \%$ showed no rebound; $80.2 \%$ of the patients with non-specific abdominal pain showed no defense, and $84.2 \%$ of them showed no rebound. Therefore, not all patients develop defense and peritoneal irritation, even in cases where surgical diagnoses are made.

Eighty-four of our patients were discharged from the ED, while 16 were hospitalized at the department; 12 underwent surgery. Sixty-six (6.9\%) of the patients in the study by Çalışkan, were operated on urgently [4]. In the study by Yeniocak et al. [5], 24 patients underwent surgery after admission to the ED.

In our study, lactate levels were found to be normal in 54 patients, and high in 31 patients. In a study by Kavakli et al. [6] into acute appendicitis, white blood cell and lactate levels were compared among 36 patients with histopathologically diagnosed acute appendicitis. In the same study, lactate levels were found to be high, with $53 \%$ specificity and $80 \%$ sensitivity. In a study by Tanaka et al. [7] involving 40 cases of bowel strangulation, lactate levels were high-sensitivity was $82 \%$ and specificity was $88 \%$-and there was a significant association between lactate level and bowel strangulation. In another study by Verma et al. [8], 20 of 50 patients received a medical diagnosis not requiring surgery, while 30 required surgery. The two groups did not differ in terms of lactate levels, and the lactate level was high in both groups. In another study 
by Lange et al., [9] lactate levels were compared among 85 patients with mesenteric ischemia, acute pancreatitis, and extensive bacterial peritonitis. It was concluded that the lactate level is related to quality of life, as well as life expectancy, and that it can indicate the need for urgent operations. In the case of mesenteric ischemia specifically, the sensitivity was $100 \%$ and the specificity was $42 \%$. Verma I et al estimated the lactate levels in peritoneal fluid and blood at the same time in the patients presented to the Ed with acute abdominal pain and they found that cutt-of lactate values were at very high sensitivity and specificity [8].

Within our group of patients, none were diagnosed with a mesenteric ischemic condition; one of the 31 patients whose lactate level was high was diagnosed with inflammatory bowel disease; one was diagnosed with acute appendicitis, two with herniation and obstruction, and nine with gastroenteritis. The lactate level of the patient with pancreatitis was normal. The low number of patients in our group means that our data are insufficient, and that we observed only a narrow range of diseases.

Limitations of our study were the less number of the total and subgroup of the patients. The insufficient number of the subgroups including severity illness affect the statistical analyses so we couldn't get valuable results.

In conclusion, we found no significant association between neither lactate levels and diagnoses nor lactate levels and the vital signs at the admission of the patients presenting to the ED with abdominal pain. To collaborate these results, it will be necessary to perform studies in multicenters on larger groups over a longer period of time.

\section{CONFLICT Of INTEREST STATEMENT}

The authors have no conflicts of interest to declare.

\section{ne) REFERENCES Cem}

[1] Navarro FJA, Tarraga LPJ, Rodriguez MJA et al. Validity of tests performed to diagnose acute abdominal pain in patients admitted at an emergency department. Rev Esp Enferm Dig 2009, 101(9):610-618.

[2] Nagurney JT, Brown DF, Chang Y et al. Use of diagnostic testing in the emergency department for patients presenting with non-traumatic abdominal pain. The Journal of emergency medicine 2003, 25(4):363-371.

[3] Chen EH, Shofer FS, Dean AJ et al. Derivation of a clinical prediction rule for evaluating patients with abdominal pain and diarrhea. The American journal of emergency medicine 2008, 26(4):450-453.

[4] Çalışkan M, Coşkun A, Acar A, et al. Multivariate prospective evaluation of patients admitted with acute abdominal pain in emergency surgery clinics. JAEM 2010, 9:75-82.
[5] Yeniocak S, Türkmen S, Uzun Ö et al.Analysis of patients presenting to the emergency department with acute abdominal pain. Journal of Academic Emergency Medicine 2012, 11(4):212.

[6] Kavakli HS, Altintas ND, Cevik Y et al. Diagnostic value of lactate levels in acute appendicitis. J Pak Med Assoc 2010, 60(11):913-915

[7] Tanaka K, Hanyu N, lida T et al. Lactate levels in the detection of preoperative bowel strangulation. Am Surg 2012, 78(1):86-88.

[8] Verma I, Kaur S, Goyal S et al. Diagnostic value of lactate levels in acute abdomen disorders. Indian J Clin Biochem 2014, 29(3):382-385.

[9] Lange $H$, Toivola A: Warning signals in acute abdominal disorders. Lactate is the best marker of mesenteric ischemia. Lakartidningen 1997, 94(20):1893-1896. 